\title{
Frontal Anaplastic Oligodendroglioma Showing Multi-organ Metastases After a Long Clinical Course
}

\author{
-Case Report-
}

\author{
Takeo UzUKA, Akiyoshi KAKITA*, Chikanori InENAGA**, Hideaki TAKAHASHI, \\ Ryuichi TANAKA, and Hitoshi TAKAHASHI**
}

Departments of Neurosurgery, * Pathology Neuroscience (Resource Branch for Brain Disease Research CBBR), and ${ }^{* *}$ Pathology, Brain Research Institute, Niigata University, Niigata

\begin{abstract}
A 17-year-old woman presented with an anaplastic oligodendroglioma manifesting as generalized seizure. Neuroimaging studies revealed a right frontal tumor. Histological examinations of biopsy specimens revealed that the tumor was oligodendroglial in nature. Total resection was repeated four times, and malignant change was evident within the tissues. The final diagnosis was anaplastic oligodendroglioma. Despite irradiation, combination chemotherapy, and interstitial hyperthermia, the tumor grew rapidly but was confined to the cavity created by previous removal operations. She suffered bone pain in the last 3 months of her life, when neuroimaging examinations disclosed multiple bone lesions. She died at the age of 29 years. At autopsy, generalized metastases from the tumor were identified at various sites, including the dura mater covering the frontal lobes and thoracic cord, cavernous sinus, tuberculum sellae, spleen, liver, pancreas, lungs, paratracheal lymph nodes, vertebral bodies, ribs, sternum, pelvis, dorsal root ganglia, and iliopsoas muscle. This rare case of cerebral anaplastic oligodendroglioma developed in adolescence, and rapid hematogenous spread of the glioma cells into the systemic organs occurred after a relatively long clinical course.
\end{abstract}

Key words: oligodendroglioma, metastasis, visceral organs, autopsy

\section{Introduction}

Oligodendroglioma is a tumor of central neuroepithelial origin, accounting for about $5 \%$ of all intracranial gliomas. ${ }^{5}$ Oligodendroglioma is mostly identified in adults, the highest incidence occurring in the fifth and sixth decades of life, although not uncommon in childhood and adolescence. ${ }^{5}$ Various types of combination chemotherapy have improved the survival of patients with oligodendroglioma and mixed oligoastrocytoma. ${ }^{2)}$

Extracranial metastasis of central nervous system (CNS) tumors is rare, with only $32(0.4 \%)$ of 8,000 patients with neuroectodermal tumors. ${ }^{14)}$ Most extracranial metastases from CNS tumors were glioblastoma multiforme or medulloblastoma, ${ }^{11-14)}$ with few documented cases of metastasis from oligodendroglioma. . $^{1,4,6-8,10)}$

Recently, we examined an autopsy case of

Received April 4, 2006; Accepted January 15, 2007 anaplastic oligodendroglioma in a young woman who had multiple organ metastases at the end stage of a relatively long disease course.

\section{Case Report}

A 29-year-old female first suffered generalized seizure in 1991 at age 17 years. Computed tomography (CT) revealed a low density mass with no calcification in the right frontal lobe involving the motor area. Magnetic resonance (MR) imaging showed the tumor as hypointense on $\mathrm{T}_{1}$-weighted images and hyperintense on $\mathrm{T}_{2}$-weighted images, with no apparent gadolinium enhancement. Gamma knife therapy was performed at a local hospital in July 1992, because the patient refused to undergo surgery, under a tentative diagnosis of glioma. She was admitted to Niigata University Hospital because of convulsions in August 1996. CT disclosed slight enlargement of the mass lesion with accompanying intratumoral hemorrhage (Fig. 1A). She received 50 Gy irradiation, and the size of the mass was reduced. 

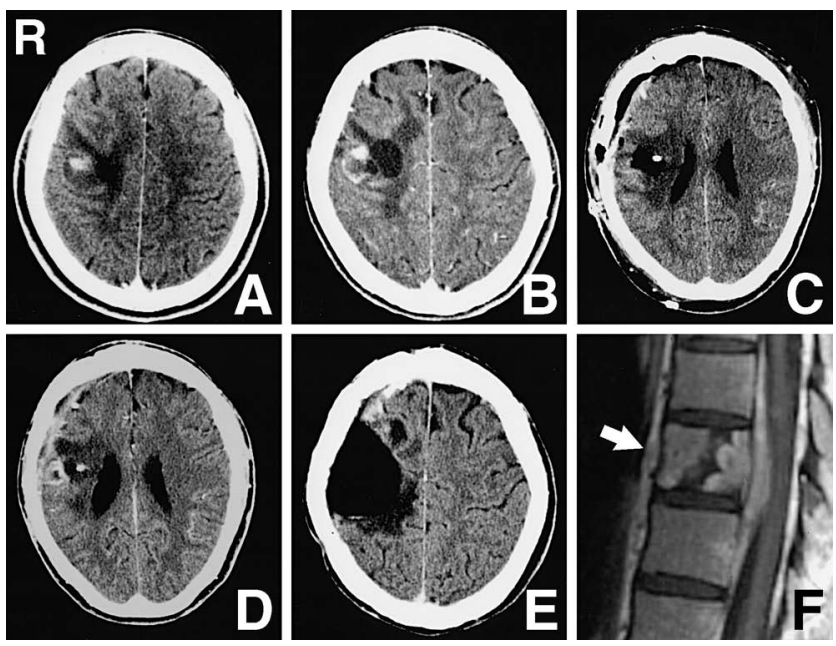

Fig. 1 A-E: Axial computed tomography scans, taken on admission (A) showing a right frontal tumor with intratumoral hemorrhage, at 4-year follow up (B) showing recurrence of the enhanced tumor with cyst, after combination chemotherapy and interstitial hyperthermia and three total resections (C) showing no apparent enhanced lesion, 1 month later (D) showing a small enhanced mass on the cavity wall, and 1 month prior to death (E) showing an additional low density lesion with slight enhancement of the wall in the anterior portion of the removal cavity. F: Sagittal spinal $T_{1}$-weighted magnetic resonance image after gadolinium infusion showing abnormal intensity in the T-10 vertebral body (arrow), and a mass compressing the cord.

The patient was followed up as an outpatient for the next 4 years. Follow-up CT disclosed regrowth of the tumor in August 2000 (Fig. 1B).

Needle biopsy was performed. Histological examination of the surgical specimens disclosed small round cells with hyperchromatic nuclei and a perinuclear halo (Fig. 2A). The histological diagnosis was anaplastic oligodendroglioma. Despite combination chemotherapy with procarbazine, ranimustine, and vincristine followed by interstitial hyperthermia, the tumor grew rapidly with multiple cysts up to February 2003. Total resection of the tumor was performed four times, and each time the histological features of the tumor tissue were anaplastic oligodendroglioma. Malignant change was evident within the specimens, including increased nuclear pleomorphism and enlargement, mitotic activity, cell density (Fig. 2B), vascular endothelial proliferation (Fig. 2C), and trabecular cell arrangement (Fig. 2D). The MIB-1 labeling index was evaluated by immunostaining with a mouse monoclonal antibody against Ki-67 antigen
(MIB-1; Immunotech, Marseilles, France; 1:100) as $52.3 \%, 56.0 \%, 81.3 \%$, and $75.0 \%$ for the first to fourth resections, respectively.

CT following the third removal showed no apparent enhanced lesion (Fig. 1C), but 1 month later follow-up CT disclosed a newly developed enhanced lesion close to the resection cavity (Fig. 1D). The last brain CT taken 1 month prior to death, in July 2003, showed an additional low density lesion (Fig. 1E). The patient developed multiple bone pains in May 2003. Photoscintigraphy demonstrated abnormally increased uptake in the bilateral shoulder joints, sternum, and bilateral iliac bones. Spinal MR imaging revealed abnormal intensity in the T-10 vertebra, and an epidural mass compressed the dorsal surface of the T9-11 cord (Fig. 1F). Histological examination of the biopsy specimen taken from the right iliac bone disclosed that the lesion was a metastatic focus of the anaplastic oligodendroglioma. She received $30 \mathrm{~Gy}$ irradiation to the T9-11 cord, resulting in marked reduction of the epidural mass. However, her consciousness gradually deteriorated and respiratory disturbance progressed. She died at the age of 29 years in August 2003, after a total clinical course of 149 months. During this period, she underwent no ventriculoperitoneal shunt operations.

At autopsy, the fresh brain weighed 1,000 g, and a resection cavity with soft tumor tissue attached to the inner wall was evident in the right frontal convexity (Fig. 3A). Histological examination found a relatively small number of residual glioma cells within the nearby brain parenchymal tissue (Fig. 3D). The tumor cells distant from the original lesion showed reduced tendency for diffuse infiltration. In contrast, numerous metastatic foci of tumor cells were found within the cranial cavity and visceral organs, including the dura mater covering the bilateral frontal lobes, cavernous sinus, sella turcica, spleen (Fig. 3B), liver, pancreas, vertebral bodies, spinal dura mater, dorsal root ganglia, ribs, sternum, pelvis (Fig. 3C), lungs (Fig. 3E), paratracheal lymph nodes, and iliopsoas muscle. Multiple vessels encasing the tumor cells were also evident in the lungs and iliopsoas muscle.

The tumor cells in most of the metastatic lesions had immature morphological features, including small nuclei, scant cytoplasm, and blurred cytoplasmic clearing, and oligodendrocytic features in some lesions. Immunohistochemistry showed no labeling of the tumor cells within the surgical specimens or autopsy materials using monoclonal antibodies against cytokeratin (CAM 5.2; Becton Dickinson, San Jose, Calif., U.S.A.; 1:5), epithelial membrane antigen (EMA; Dako, Glostrup, Denmark; 1:100), leukocyte common antigen (LCA; Dako; 1:50), CD20 (clone L26; Dako; 1:100), neurofilament polypeptides (70 and $200 \mathrm{kDa}$; Sanbio BV, Uden, The Netherlands; 1:50), synaptophysin (a gift from Dr. K. Obata); 1:1,000), phosphorylated or non-phosphorylated 


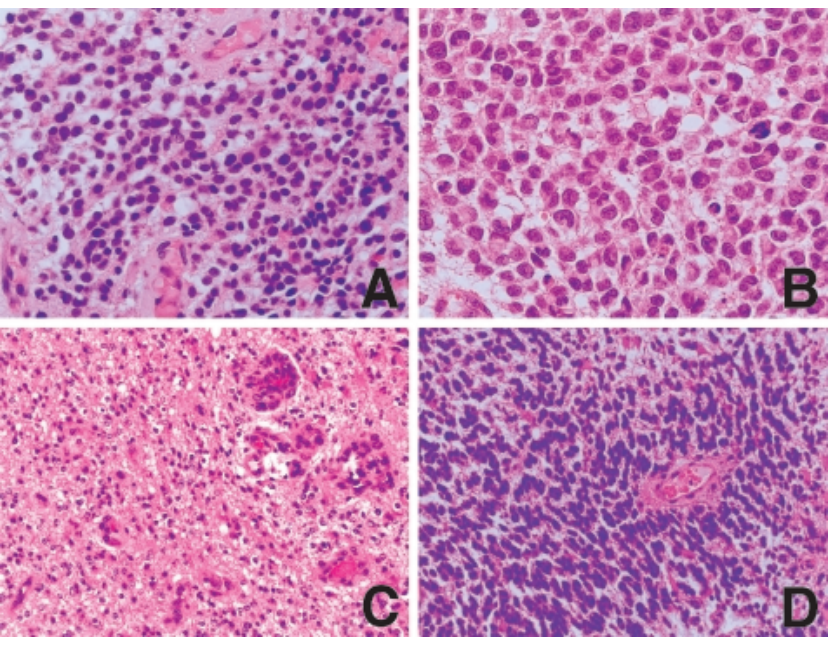

Fig. 2 Photomicrographs of the tumor biopsy specimens, from the first biopsy (A) showing oligodendroglial cells with moderate cellularity, and after the first total resection (B) showing nuclear pleomorphism and enlargement, and scattered mitotic figures, the second (C) showing vascular endothelial proliferation, and the third (D) showing trabecular arrangement with amorphous extracellular spaces. Hematoxylin and eosin stain, A: $\times 400$, B: $\times 400, \mathrm{C}: \times 200$, D: $\times 200$.

epitopes of neurofilament (SMI-36 or SMI-32, respectively; Sternberger Monoclonal, Lutherville, Md., U.S.A.; 1:1,000), and neuronal nuclei (Neu-N; Chemicon, Temecula, Calif., U.S.A.; 1:100). On the other hand, a large proportion of the tumor cell nuclei was labeled with polyclonal antibody against Olig-2 (Immuno-Biological Laboratories Co., Ltd., Fujioka, Gunma; 1:200) (data not shown). None of the tumor lesions had developed primarily in the visceral organs.

\section{Discussion}

The present case was a rare example of anaplastic oligodendroglioma that originally developed in the frontal lobe of a young subject, followed by generalized metastases to many organs at the end stage of a long clinical course. The complete autopsy of this patient met Weiss's criteria for confirming visceral lesions as metastases from CNS tumor, ${ }^{15)}$ based on histological examinations.

Over 30 cases of metastases from oligodendroglioma have been reported. ${ }^{1,4,6-8,10)}$ The bones, especially the vertebrae, are the sites most frequently involved, ${ }^{10)}$ in contrast to glioblastoma, which most often shows metastasis to the lungs and lymph nodes, followed by the bones, liver, and pleura. ${ }^{5)}$ Anaplastic oligodendroglioma could develop
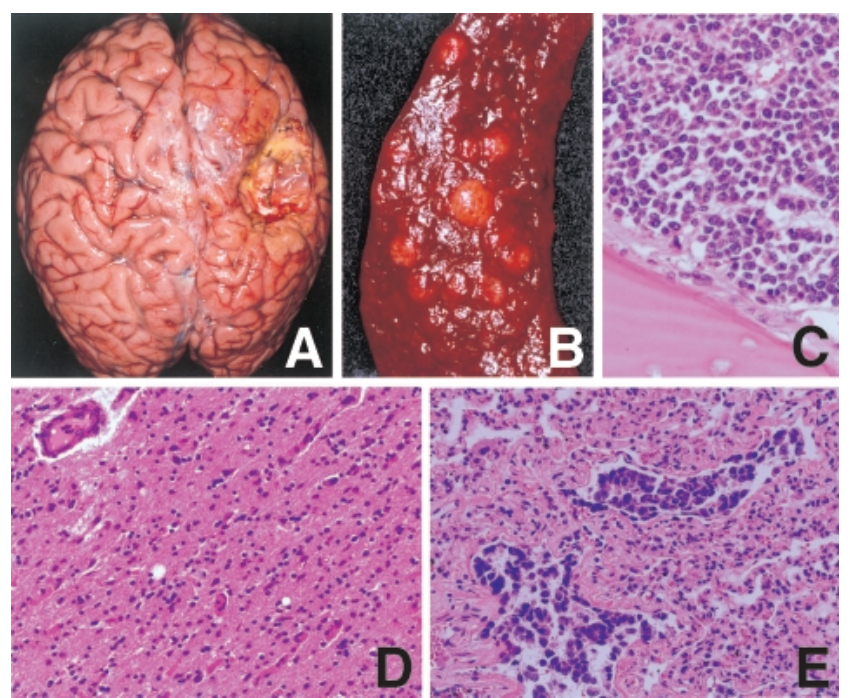

Fig. 3 A, B: Photographs of the brain (A) showing the resection cavity in the right frontal lobe, and the spleen (B) showing multiple nodular metastatic foci. C-E: Photomicrographs of the iliac bone (C) demonstrating metastatic foci, the residual tumor in the brain parenchymal tissue (D) showing cells with small, hyperchromatic nuclei, and scant cytoplasm, and the metastatic focus of oligodendroglioma in the lung (E). Hematoxylin and eosin stain, C: $\times 200, \mathrm{D}: \times 200, \mathrm{E}: \times$ 200.

metastases more frequently than glioblastoma or anaplastic astrocytoma, so the true frequency of metastases from oligodendrogliomas remains unclear. ${ }^{4)}$ These differences are presumably attributable to differences in patient selection criteria, protocols used for determining therapy and survival periods, and the criteria used for histological diagnosis. ${ }^{5)}$ More recently, review of a series of oligodendrogliomas with metastases found that the median survival was 32 months, ${ }^{1}$ which is apparently shorter than that for patients with oligodendroglioma without metastases of 45.5 months. ${ }^{6)}$ The present patient survived for approximately 150 months, which should be regarded as an exceptionally long clinical course.

Neurosurgical procedures, including craniotomy and ventriculoperitoneal shunt placement, carry the risk of extraneural metastases. ${ }^{1,3,13)}$ The great majority of well-documented cases of glioblastoma multiforme with extraneural metastases (237 of 247 cases) were associated with such procedures. ${ }^{3)}$ Surgical procedures may expose the tumor tissue to the lymphatic system outside the nervous system. Furthermore, breaching of the intracerebral vasculature certainly introduces the risk of tumor cell seeding. In addition, tumor cells can spread directly through the cerebrospinal fluid. In the present case, 
two biopsies and four removal operations were performed, which might have been at least partly responsible for the widespread metastases. Considering the clinicopathological features of the present case, the rapid hematogenous spread probably occurred during the last 3 months of the disease course. The last operation had been performed 3 months before the patient first began to suffer bone pain. In addition, another metastatic route through the vertebral venous system (Batson's plexus) is possible, because of the presence of the spinal epidural mass.

Throughout the course, the tumor grew in a relatively restricted portion of the brain, occupying the cavity created by the previous resection rather than infiltrating widely within the brain. The histological features of the resected specimens and the residual tumor in the autopsied brain were compatible with those of anaplastic oligodendroglioma. ${ }^{5)}$ Immunohistochemical profiles of the cells for several neuronal and lymphocytic markers were compatible with this diagnosis. Malignant transformation of the tumor cells seems to be one of the crucial factors for metastasis, even in intracranial neuroepithelial tumors. Cells in metastatic foci from gliomas tend to have greater histologic anaplasia than the primary tumor. ${ }^{5)}$ In the present case, the surgical specimens showed features indicating malignant transformation including microvascular proliferation, frequent mitotic figures, and immature morphology of the tumor cells. The MIB-1 labeling index showed a tendency to increase, further demonstrating the biological changes of the tumor. Therapeutic strategies including radiation and chemotherapy may also influence the biological behavior of the tumor cells. The present patient first received radiation therapy, combination chemotherapy after 6 years, and surgical resection 1 year later. The cellular or molecular mechanisms underlying the metastasis of glioma cells remain to be elucidated.

Chemotherapy is a common choice of treatment for metastases. One case of bone metastasis from anaplastic oligodendroglioma showed a dramatic response to temozolomide. ${ }^{7}$ ) We performed palliative radiation therapy for the spinal epidural mass, leading to marked reduction in the size of the lesion. Therefore, irradiation may be another choice of treatment for such lesions. Metastases from cerebral oligodendroglioma are very rare, but the present case suggests that prompt diagnosis and irradiation may be effective in increasing survival.

\section{Acknowledgments}

We wish to express our appreciation to Ms. C. Tanda, Ms. J. Takasaki, and Ms. N. Kaneko for technical assistance.

\section{References}

1) Dawson TP: Pancytopaenia from a disseminated anaplastic oligodendroglioma. Neuropathol Appl Neurobiol 23: 516-520, 1997

2) Glass J, Hochberg FH, Gruber ML, Louis DN, Smith $\mathrm{D}$, Rattner B: The treatment of oligodendrogliomas and mixed oligodendroglioma-astrocytomas with PCV chemotherapy. J Neurosurg 76: 741-745, 1992

3) Huang P, Allam A, Taghian A, Freeman J, Duffy M, Suit HD: Growth and metastatic behavior of five human glioblastomas compared with nine other histological types of human tumor xenografts in SCID mice. J Neurosurg 83: 308-315, 1995

4) Macdonald DR, O'Brien RA, Gilbert JJ, Cairncross JG: Metastatic anaplastic oligodendroglioma. Neurology 39: 1593-1596, 1989

5) Mclendon R, Enterline DS, Tien RD, Thorstad WL, Bruner JM: Tumors of central neuroepithelial origin, in Bigner DD, McLendon RE, Bruner JM (eds): Russel and Rubinstein's Pathology of Tumors of the Nervous System, vol 1, ed 6. London, Arnold, 1998, pp 307-571

6) Mork SJ, Lindegaard KF, Halvorsen TB, Lehmann EH, Solgaard T, Hatlevoll R, Harvei S, Ganz J: Oligodendroglioma: incidence and biological behavior in a defined population. J Neurosurg 63: 881-889, 1985

7) Morrison T, Bilbao JM, Yang G, Perry JR: Bony metastases of anaplastic oligodendroglioma respond to temozolomide. Can J Neurol Sci 31: 102-108, 2004

8) Nakamura $O$, Watanabe T, Nomura K, Nakajima T: [Diffuse bone marrow metastasis of an anaplastic oligodendroglioma]. No Shinkei Geka 13: 903-909, 1985 (Jpn, with Eng abstract)

9) Obata K, Kojima N, Nishiye H, Inoue H, Shirao T, Fujita SC, Uchizono K: Four synaptic vesicle-specific proteins; identification by monoclonal antibodies and distribution in the nervous tissue and the adrenal medulla. Brain Res 404: 169-179, 1987

10) Ordonez NG, Ayala AG, Leavens ME: Extracranial metastases of oligodendroglioma: report of a case and review of the literature. Neurosurgery 8: 391-396, 1981

11) Pasquier B, Pasquier D, N'Golet A, Panh MH, Couderc P: Extraneural metastases of astrocytomas and glioblastomas: clinicopathological study of two cases and review of literature. Cancer 45: 112-125, 1980

12) Rickert $\mathrm{CH}$ : Extraneural metastases of paediatric brain tumours. Acta Neuropathol (Berl) 105: 309-327, 2003

13) Schweitzer T, Vince GH, Herbold C, Roosen K, Tonn JC: Extraneural metastases of primary brain tumors. J Neurooncol 53: 107-114, 2001

14) Smith DR, Hardman JM, Earle KM: Metastasizing neuroectodermal tumors of the central nervous system. J Neurosurg 31: 50-58, 1969

15) Weiss L: A metastasizing ependymoma of the cauda equina. Cancer 8: 161-171, 1955

Address reprint requests to: Takeo Uzuka, M.D., Department of Neurosurgery, Brain Research Institute, Niigata University, 1 Asahimachi-dori, Niigata 951-8585, Japan.

e-mail: takeo@bri.niigata-u.ac.jp 